Supplement of

\title{
Identification and validation of key miRNAs and miRNA-mRNA regulatory network associated with uterine involution in postpartum Kazakh sheep
}

Heng Yang et al.

Correspondence to: Heng Yang (yh20183007@swu.edu.cn) and Huihao Xu (xuhuihao2dai@163.com)

The copyright of individual parts of the supplement might differ from the article licence. 
Table S1A the primer sequence of miRNA (hypothalamus)

\begin{tabular}{ccc}
\hline & miR_name & Primer \\
\hline & oar-miR-200a & GGGGTAACACTGTCTGGTAACGATGTT \\
oar-miR-200b & GGGGTAATACTGCCTGGTAATGATG \\
oar-miR-200c & GGTAATACTGCCGGGTAATGATGGA \\
UFH/USH & oar-miR-665-3p & CGGGCCATAATACATGGTTAACC \\
& oar-miR-362 & GCAATCCTTGGAACCTAGGTGT \\
& oar-novel-miR-1109-5p & GGCCCGTCCCGTGCGTCAA \\
& oar-novel-miR-881-5p & CTGGCTCCGTGTCTTCACTCC \\
& U6 & CAAGGATGACACGCAAATTCG \\
\hline
\end{tabular}

Table S1B the primer sequence of miRNA (uterus)

\begin{tabular}{ccc}
\hline miR_name & Primer \\
\hline oar-miR-200a & GGGGTAACACTGTCTGGTAACGATGTT \\
oar-miR-99a & CCGAACCCGTAGATCCGATCTTG \\
oar-miR-133 & TCCGTTTTGGTCCCCTTCAAC \\
oar-miR-379-5p & CGTGGTAGACTATGGAACGTAGGC \\
& oar-novel-miR-1185-3p & CCCGGCCTGGAATGTAAAGAAGTATGTAT \\
oar-novel-miR-1109-5p & GGCCCGTCCCGTGCGTCAA \\
U6 & CAAGGATGACACGCAAATTCG \\
\hline
\end{tabular}

Table S1C the primer sequence of target genes

\begin{tabular}{cccc}
\hline & Gene & Primer $\left(5^{\prime}-3^{\prime}\right)$ & Product size $(\mathrm{bp})$ \\
\hline \multirow{3}{*}{ PTEN } & F: CACACGACGGGAAGACAAGT & 167 \\
& & R: AGGTTTCCTCTGGTCCTGGTA & \\
UF/US & FGFR1 & F: CAAACCAAACCGTATGCCCG & 192 \\
& & R: ACCTTGTAGCCTCCGATCCT & \\
& F: GGGAGTCCGCATGGAAGAAA & 122 \\
\hline
\end{tabular}

